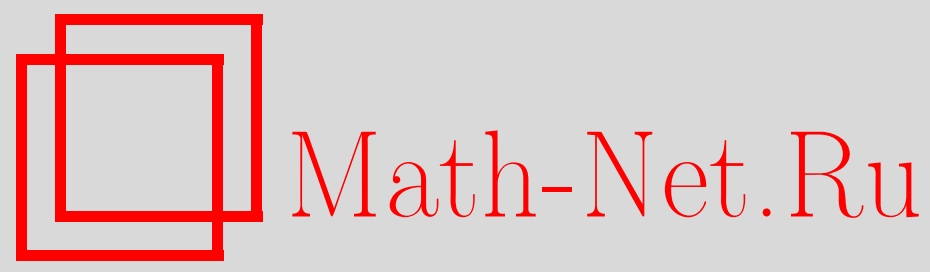

А. М. Ахтямов, О коэффициентах разложений по собственным функциям краевых задач с параметром в граничных условиях, Матем. заметки, 2004, том 75, выпуск 4, 493-506

DOI: https://doi.org/10.4213/mzm47

Использование Общероссийского математического портала Math-Net.Ru подразумевает, что вы прочитали и согласны с пользовательским соглашением http://www.mathnet.ru/rus/agreement

Параметры загрузки:

IP : 52.87 .193 .239

26 апреля 2023 г., 15:57:51 


\section{О КОЭФФИЦИЕНТАХ РАЗЛОЖЕНИЙ \\ ПО СОБСТВЕННЫМ ФУНКЦИЯМ КРАЕВЫХ ЗАДАЧ \\ С ПАРАМЕТРОМ В ГРАНИЧНЫХ УСЛОВИЯХ}

\section{А. М. Ахтямов}

Рассматриваются краевые задачи для обыкновенного дифференциального уравнения $n$-го порядка с полиномиальньп вхождением спектрального параметра в уравнение и краевые условия. Для шшрокого класса таких задач найдены коэффициенты разложений в ряд по системе собственных и присоединенных функций линеаризатора Шкаликова.

Библиограффия: 16 названий.

Рассмотрим следующую спектральную задачу

$$
\begin{aligned}
l(y, \lambda) & =l_{0}(y)+\lambda l_{1}(y)+\cdots+\lambda^{n} l_{n}(y)=0 \\
U_{j}(y, \lambda) & =U_{j}^{0}(y)+\lambda U_{j}^{1}(y)+\cdots+\lambda^{\nu_{j}} U_{j}^{\nu_{j}}(y)=0, \quad j=1,2, \ldots, n .
\end{aligned}
$$

Здесь

$$
\begin{aligned}
l_{\nu}(y) & =\sum_{s=\nu}^{n} p_{\nu s}(x) y^{(n-s)}, \quad \nu=0,1, \ldots, n, \\
U_{j}^{\nu}(y) & =\sum_{k=0}^{n-1} a_{j \nu k} y^{(k)}(0)+\sum_{k=0}^{n-1} b_{j \nu k} y^{(k)}(1), \quad \nu=0,1, \ldots, \nu_{j} .
\end{aligned}
$$

Коэффициенты $a_{j \nu k}, b_{j \nu k}-$ комплексные числа, которые, в частности, могут быть равны нулю, $p_{s s}(x)=$ const, $s=1,2, \ldots, n, p_{n n} \neq 0, p_{\nu s}(x)$ - достаточно гладкие функции, а $a_{j k}(\lambda), b_{j k}(\lambda)$ - произвольные полиномы от $\lambda$.

Изучение краевых задач вида (1), (2) ведет начало от работ Биркгофа [1], [2]. В случае, когда $l_{s}(y)=0$ при $s=1,2, \ldots, n-1, p_{00}=1$ и $p_{0 k}=0$ при $k=1,2, \ldots, n-1$, в этих работах была найдена фундаментальная система решений уравнения (1) и выделен класс регулярных краевых условий, для которого была получена теорема о разложениях гладких функций в ряды по собственным и присоединенным функциям задачи.

В [3] была найдена асимптотика (по $\lambda$ ) системы фундаментальных решений уравнения (1) и получена теорема о возможности разложения гладкой функции в ряд по собственньм функциям задачи (1), (2) в самом общем случае.

Работа выполнена при поддержке Российского фонда фундаментальных исследований, грант № 01-01-00996, и Министерства образования России, грант Е02-1.0-77. 
В фундаментальных работах Келдьша [4], [5] было показано, что запас собственных и присоединенных функций полиномиального пучка $n$-го порядка столь богат, что в ряды по этим функциям, как правило, могут быть разложены одновременно $n$ функций. Кроме того, Келдьш отметил, что постановка задачи об $n$-кратных разложениях возникает естественным образом для обоснования метода Фурье при решении начально-краевой задачи для соответствуюшего уравнения с частными производными, из которого спектральная задача получается после разделения переменных.

В работе [6] были построены операторы, линеаризующие задачу (1), (2) в пространствах $\mathscr{W}_{2, U}^{r} \times \mathbb{C}^{N_{r}}$, которые являются подпространствами конечной коразмерности в произведениях пространств $W_{2}^{n+r-1} \times \cdots \times W_{2}^{r} \times \mathbb{C}^{N_{r}}$. Эти пространства в [6] построены явно. При этом если $r \geqslant r_{0}=\max (0, \rho-n+1)$, где $\rho$ - максимальньй из порядков краевых условий спектральной задачи $(1),(2)$, то $N_{r}=0$ и линеаризатор имеет вид

$$
H\left\{v_{0}, v_{1}, \ldots, v_{n-1}\right\}=\left\{v_{1}, v_{2}, \ldots, v_{n-1},-p_{n n}^{-1}\left(l_{0}\left(v_{0}\right)+l_{1}\left(v_{1}\right)+\cdots+l_{n-1}\left(v_{n-1}\right)\right)\right\} .
$$

При условии регулярности задачи (1), (2) в [6] доказана теорема о базисности Рисса собственных и присоединенных функций $\tilde{\mathbf{v}}_{k}^{h}$ линеаризатора $H$. А именно, любой элемент пространства $\mathscr{W}_{2, U}^{r}$ может быть разложен в ряд

$$
\tilde{\mathbf{v}}=\sum_{k=1}^{\infty} \sum_{h=0}^{p_{k}} c_{k h} \cdot \tilde{\mathbf{v}}_{k}^{h}
$$

где ряд безусловно сходится после заключения некоторых его членов в скобки, расстановка которых не зависит от $\tilde{\mathbf{v}}$.

Важной является задача вычисления $c_{k h}$ разложения элемента $\tilde{\mathbf{v}} \in \mathscr{W}_{2, U}^{r}$ в ряд по системе $\tilde{\mathbf{v}}_{k}^{h}$. Конечно, $c_{k h}=\left\langle\tilde{\mathbf{v}}, \tilde{\mathbf{g}}_{k}^{h}\right\rangle$, где $\left\{\tilde{\mathbf{g}}_{k}^{h}\right\}$ - система функций, биортогональная к $\left\{\tilde{\mathbf{v}}_{k}^{h}\right\}$. Но вычислить явно $\left\{\tilde{\mathbf{g}}_{k}^{h}\right\}-$ трудно осуществимая задача. Мы же хотим выразить коэффициенты разложений в терминах собственных функций задачи, сопряженной $\mathrm{K}(1),(2)$.

Если краевые условия (2) не зависят от $\lambda$, то задачу $(1),(2)$ можно рассматривать как пучок $n$-го порядка дифференциальных операторов в $L_{2}(0,1)$ :

$$
\left(P_{0}+\lambda P_{1}+\cdots+\lambda^{n} P_{n}\right) y=0,
$$

где области определения операторов $P_{\nu}$ можно считать совпадающими с

$$
D\left(P_{0}\right)=\left\{y: y \in W_{2}^{2}, U_{j}(y)=0, j=1,2, \ldots, n\right\} .
$$

В этом случае формулы для коэффициентов разложения получаются из общих формул [7, теорема 2.1].

Если краевые условия (2) зависят от $\lambda$, то область определения пучка операторов также зависит от $\lambda$ и формулы из [7] непосредственно применены быть не могут.

В этой работе мы выделим класс задач вида (1), (2), для которых мы предложим два метода для вычисления коэффициентов разложения. Полученные формулы будут иметь разную форму. Если система $\left\{\tilde{\mathbf{v}}_{k}^{h}\right\}$ собственных и присоединенных функций линеаризатора $H$ полна в пространстве, где действует этот оператор (например, это так, если задача регулярна), то биортогональная система определяется однозначно, а потому значения коэффициентов разложения, даваемые разными формулами, будут совпадать. 
Предположим, что формы $U_{1}^{0}(y), U_{2}^{0}(y), \ldots, U_{n}^{0}(y)$ задачи $(1),(2)$ линейно независимы (этого всегда можно добиться, рассматривая соответствующие линейные комбинации форм $\left.U_{j}(y, \lambda)\right)$. Обозначим через

$$
U_{n+1}^{0}(y), U_{n+2}^{0}(y), \ldots, U_{2 n}^{0}(y)
$$

$n$ линейных однородных форм от переменных $y^{(k)}(0), y^{(k)}(1), k=0,1, \ldots, n-1$, с коэффициентами, не зависящими от $\lambda$, такие, что формы

$$
U_{1}^{0}(y), U_{2}^{0}(y), \ldots, U_{n}^{0}(y), U_{n+1}^{0}(y), U_{n+2}^{0}(y), \ldots, U_{2 n}^{0}(y)
$$

образовывали бы линейную независимую систему.

На протяжении всей работы через $\langle\cdot, \cdot\rangle$ Н обозначается скалярное произведение в гильбертовом пространстве $\mathscr{H}$.

Пусть $l_{0}^{*}(z)$ - дифференциальное выражение, сопряженное к выражению $l_{0}(y)$ в пространстве $L_{2}$, определяемое интегрированием по частям:

$$
\left\langle l_{0}(y), z\right\rangle_{L_{2}}=\left\langle y, l_{0}^{*}(z)\right\rangle_{L_{2}}+P_{0}(y, z) \text {. }
$$

Через $V_{1}^{0}(z), \ldots, V_{n}^{0}(z), V_{n+1}^{0}(z), \ldots, V_{2 n}^{0}(z)$ обозначим линейные однородные формы от переменных $z^{(k)}(0), z^{(k)}(1), k=0,1, \ldots, n-1$, которые содержатся в билинейной форме обьнтегрированных членов

$$
P_{0}(y, z)=U_{1}^{0}(y) \cdot \overline{V_{2 n}^{0}(z)}+U_{2}^{0}(y) \cdot \overline{V_{2 n-1}^{0}(z)}+\cdots+U_{2 n}^{0}(y) \cdot \overline{V_{1}^{0}(z)}
$$

В конце статьи приводится основной результат работы - теорема о вычислении коэффициентов разложения (3) для спектральных задач $(1),(2)$, удовлетворяющих следующим условиям $\mathbf{U}$ и $\mathbf{P}$.

УСловиЕ $\mathbf{U}$. Формы $U_{j}^{\nu}(y)$ при $\nu=1,2, \ldots, m$ являются линейньми однородными формами от переменных $U_{n+1}^{0}(y), U_{n+2}^{0}(y), \ldots, U_{2 n}^{0}(y)$ :

$$
U_{i}^{\nu}(y)=\xi_{i 1}^{\nu} U_{n+1}^{0}(y)+\xi_{i 2}^{\nu} U_{n+2}^{0}(y)+\cdots+\xi_{i n}^{\nu} U_{2 n}^{0}(y)
$$

где $\xi_{i j}^{\nu} \in \mathbb{C}, i, j=1,2, \ldots, n$.

(Постоянные $\xi_{i j}^{\nu}$ могут быть равны нулю. В частности, если краевые условия не зависят от $\lambda$, то условие $\mathbf{U}$ вьполняется, поскольку можно выбрать все $\xi_{i j}^{\nu}$ равньми нулю).

УсловиЕ Р. Билинейные формы обынтегрированных членов $P_{\nu}(y, z), \nu=1,2$, $\ldots, n$, которые возникают из равенства

$$
\left\langle l_{\nu}(y), z\right\rangle_{L_{2}}=\left\langle y, l_{\nu}^{*} z\right\rangle_{L_{2}}+P_{\nu}(y, z)
$$

удовлетворяют условию

$$
P_{\nu}(y, z)=\overline{\gamma_{1}^{\nu}} \cdot U_{n+1}^{0}(y)+\overline{\gamma_{2}^{\nu}} \cdot U_{n+2}^{0}(y)+\cdots+\overline{\gamma_{n}^{\nu}} \cdot U_{2 n}^{0}(y), \quad \nu=1,2, \ldots, n
$$

где коэффициенты $\overline{\gamma_{1}^{\nu}}, \overline{\gamma_{2}^{\nu}}, \ldots, \overline{\gamma_{n}^{\nu}}$ - некоторые линейные однородные функции от переменных $z(0), z(1), z^{\prime}(0), z^{\prime}(1), \ldots, z^{(n-1)}(0), z^{(n-1)}(1)$. 
Заметим, что условия $\mathbf{U}$ и $\mathbf{P}$ не столь ограничительны, как это может показаться на первый взгляд. В частности, эти условия вьполнены, если краевые условия не зависят от спектрального параметра. Таким образом, рассматриваемый класс охватьвает все задачи для пучков линейных дифференциальных операторов, в краевые условия которых спектральный параметр не входит. Кроме того, конкретные задачи с параметром в краевых условиях (см. [8]-[16]), рассмотренные ранее, также попадают в этот класс. В конце статьи для примера вычислены коэффициенты разложения для одной из таких задач.

Доказательство теоремы о вычислении коэффициентов проводится в два этапа. На первом этапе спектральная задача сводится к пучку неограниченных операторов, действующему в пространстве $L_{2} \times \mathbb{C}^{n}$, таким образом, чтобы между собственными функциями сопряженного пучка операторов и сопряженной спектральной задачи можно было установить взаимно однозначное соответствие. На втором этапе показьвается, что коэффищиенты разложения по производньм цепочкам пучка операторов совпадают с коэффициентами из равенства (3).

Доказательству теоремы предшествуют три леммы. В лемме 1 доказьвается существование сопряженного пучка операторов. В лемме 2 указан общий метод определения области определения сопряженного пучка операторов (или сопряженного оператора). В лемме 3 показано, что собственные значения сопряженной спектральной задачи и сопряженного пучка операторов совпадают, а между их собственными и присоединенными функциями можно установить взаимно однозначное соответствие.

Для доказательства этих результатов нам потребуются вспомогательные конструкции.

Далее через $m$ обозначаем $\max \left(n, \nu_{1}, \nu_{2}, \ldots, \nu_{n}\right)$, а через $q$ - порядок максимальной производной переменных $y^{(i)}(0), y^{(i)}(1)$, содержащейся в формах $U_{n+1}^{0}, U_{n+2}^{0}, \ldots, U_{2 n}^{0}$.

Определим в пространстве $L_{2} \times \mathbb{C}^{n}$ операторы $A_{\nu}$ следующим образом:

$$
\begin{aligned}
A_{\nu} \mathbf{y}= & \left\{l_{\nu}(y), U_{1}^{\nu}(y), U_{2}^{\nu}(y), \ldots, U_{n}^{\nu}(y)\right\} \\
D\left(A_{\nu}\right)=\{ & \left\{\mathbf{y}=\left\{y, c_{1}, c_{2}, \ldots, c_{n}\right\}: y \in W_{2}^{n},\right. \\
& \left.c_{1}=U_{n+1}^{0}(y), c_{2}=U_{n+2}^{0}(y), \ldots, c_{n}=U_{2 n}^{0}(y)\right\} .
\end{aligned}
$$

Здесь

$$
\nu=0,1, \ldots, n, n+1, \ldots, m, \quad m=\max \left(n, \nu_{1}, \nu_{2}, \ldots, \nu_{n}\right) .
$$

При $n<m$ выражение $l_{\nu}(y), \nu=n+1, \ldots, m$, считаем тождественньм нулю; а при $\nu_{j}<m$ считаем тождественно равными нулю также и формы $U_{j}^{\nu}(y), \nu=\nu_{j+1}, \nu_{j+2}$, $\ldots, m$.

Составим из операторов $A_{\nu}$ пучок операторов

$$
A(\lambda)=A_{0}+\lambda A_{1}+\cdots+\lambda^{n} A_{n}+\cdots+\lambda^{m} A_{m} .
$$

Легко показать, что

$1)$ собственные значения краевой задачи $(1),(2)$ и пучка операторов $A(\lambda)$ совпадают с учетом их кратностей;

2) каждой цепочке собственной и присоединенных функций $y^{0}, y^{1}, \ldots, y^{p_{k}}$ задачи (1), $(2)$, отвечающей собственному значению $\lambda_{0}$, соответствует цепочка собственной и присоединенных функций $\mathbf{y}^{0}, \mathbf{y}^{1}, \ldots, \mathbf{y}^{p_{k}}$ пучка $A(\lambda)$, отвечающая тому же собственному 
значению $\lambda_{0}$; при этом первые компоненты собственной (соответственно присоединенной) функции

$$
\mathbf{y}^{s}=\left\{y^{s}, U_{n+1}^{0}\left(y^{s}\right), U_{n+2}^{0}\left(y^{s}\right), \ldots, U_{2 n}^{0}\left(y^{s}\right)\right\}
$$

пучка $A(\lambda)$ совпадают с собственной (соответственно присоединенной) функцией $y^{s}$ задачи $(1),(2)$.

ЛЕмма 1 (о плотности). Области определения операторов $A_{\nu}, \nu=0,1, \ldots, m$, плотны в пространстве $L_{2} \times \mathbb{C}^{n}$.

ДокАзАТЕЛьСтво. Области определения операторов $A_{\nu}, \nu=0,1, \ldots, m$, совпадают и равны

$$
\begin{aligned}
D\left(A_{\nu}\right)=\{\mathbf{y}= & \left\{y, c_{1}, c_{2}, \ldots, c_{n}\right\}: y \in W_{2}^{n}, \\
& \left.c_{1}=U_{n+1}^{0}(y), c_{2}=U_{n+2}^{0}(y), \ldots, c_{n}=U_{2 n}^{0}(y)\right\} .
\end{aligned}
$$

Для доказательства плотности $D\left(A_{\nu}\right)$ в пространстве $L_{2} \times \mathbb{C}^{n}$ достаточно показать, что для любых чисел $\alpha_{1}, \alpha_{2}, \ldots, \alpha_{n}$, любого $\varepsilon>0$ и любой функции

$$
\mathbf{v}=\left\{v, \alpha_{1}, \alpha_{2}, \ldots, \alpha_{n}\right\} \in L_{2} \times \mathbb{C}^{n}
$$

найдется такая функция $\mathbf{y}=\left\{y, U_{n+1}^{0}(y), U_{n+2}^{0}(y), \ldots, U_{2 n}^{0}(y)\right\}$, что

$$
U_{n+i}^{0}(y)=\alpha_{i}, \quad i=1,2, \ldots, n, \quad \text { и } \quad\|v-y\|_{L_{2}}<\varepsilon .
$$

Формы $U_{n+i}^{0}(\cdot), i=1,2, \ldots, n$, линейно независимы. Значит, для любых чисел $\alpha_{1}, \alpha_{2}, \ldots, \alpha_{n}$ можно подобрать функцию $u \in W_{2}^{n}$, для которой вьполнены равенства $U_{n+i}^{0}(u)=\alpha_{i}, i=1,2, \ldots, n$.

Так как порядки форм $U_{n+i}^{0}(u), i=1,2, \ldots, n$, не превосходят $n-1$, то множество всех функций $w \in W_{2}^{n}$, удовлетворяющих краевым условиям $U_{n+i}^{0}(w)=0, i=1,2$, $\ldots, n$, всюду плотно в пространстве $L_{2}$. Поэтому любая функция из $L_{2}$ может быть приближена функцией $w$. Любая функция из $L_{2}$ может быть представлена в виде $v-u$, где $v$ - произвольная функция из $L_{2}$; следовательно, $\|(v-u)-w\|_{L_{2}}<\varepsilon$. Положив $y=u+w$, получаем, что $\|v-y\|<\varepsilon$ и $U_{n+i}^{0}(u)=\alpha_{i}, i=1,2, \ldots, n$. Лемма доказана.

СлЕДСТВИЕ. Область определения пучка операторов $A(\lambda)$ плотна в пространcmве $L_{2} \times \mathbb{C}^{n}$.

ЗАмЕчАниЕ 1. Аналогично можно показать, что линеал функций

$$
\left\{y, U_{n+1}^{0}(y), U_{n+2}^{0}(y), \ldots, U_{2 n}^{0}(y)\right\}
$$

где $y \in W_{2}^{r}, r>n$, плотен в пространстве $L_{2} \times \mathbb{C}^{n}$.

Это замечание нам понадобится в дальнейшем.

ЛЕмма 2 (о сопряженном операторе). Пусть в гильбертовом пространстве भ̈ с скалярным произведением $\langle\cdot, \cdot\rangle \mathscr{H}$ заданы операторы $H$ и $M$, образом которых является все пространство Н̈. Если оператор Н имеет плотную область определения в $\mathscr{H}$ и, кроме того, для любых әлементов $\mathbf{v} \in D(H) u \mathbf{g} \in D(M)$ выполнено равенство

$$
\langle H \mathbf{v}, g\rangle_{\mathscr{H}}=\langle\mathbf{v}, M \mathbf{g}\rangle_{\mathscr{H}}
$$

то оператор $M$ является сопряженным $к H$. 
ДокаЗАтельство. Равенство (4) означает, что $M \subseteq H^{*}$. Для доказательства того, что $M=H^{*}$, осталось доказать включение $D(M) \supseteq D\left(H^{*}\right)$. Пусть $\mathbf{r} \in D\left(H^{*}\right)$ и $H^{*} \mathbf{r}=\mathbf{h}$. Тогда для любого элемента $\mathbf{v} \in D(H)$ верно равенство

$$
\langle H \mathbf{v}, \mathbf{r}\rangle_{\mathscr{H}}=\langle\mathbf{v}, \mathbf{h}\rangle_{\mathscr{H}} .
$$

Поскольку образом оператора $M$ является все пространство $\mathscr{H}$, то для элемента $\mathbf{h}$ найдется элемент $\mathbf{r}_{1}$ такой, что $M \mathbf{r}_{1}=\mathbf{h}$. Тогда для любого элемента $\mathbf{v} \in D(H)$ верно равенство

$$
\left\langle H \mathbf{v}, \mathbf{r}_{1}\right\rangle_{\mathscr{H}}=\langle\mathbf{v}, \mathbf{h}\rangle_{\mathscr{H}} .
$$

Из (5), (6) получаем следующее равенство:

$$
\left\langle H \mathbf{v}, \mathbf{r}-\mathbf{r}_{1}\right\rangle \mathscr{H}=0 .
$$

Обозначим через $\mathbf{f}$ элемент $H \mathbf{v}$. Тогда последнее уравнение примет вид

$$
\left\langle\mathbf{f}, \mathbf{r}-\mathbf{r}_{1}\right\rangle_{\mathscr{H}}=0
$$

Равенство (7) означает, что элемент $\mathbf{r}-\mathbf{r}_{1}$ ортогонален элементу $\mathbf{f}$. Но так как $\mathbf{f}$ является произвольньм элементом пространства $\mathscr{H}$, то (7) равносильно тому, что элемент $\mathbf{r}-\mathbf{r}_{1}$ ортогонален всему пространству $\mathscr{H}$. Отсюда $\mathbf{r}-\mathbf{r}_{1}=\mathbf{0}$.

Таким образом, $\mathbf{r}=\mathbf{r}_{1} \in \mathscr{H}$ и лемма 2 доказана.

ЛЕмма 3. Пусть для каждого из коэффициентов задачи (1), (2) выполнены условия $\mathbf{U}$ и $\mathbf{P}$. Тогда собственные значения сопряженной краевой задачи и собственные значения сопряженного $\kappa A(\lambda)$ пучка операторов $[A(\bar{\lambda})]^{*}$ совпадают $c$ учетом их кратностей, а каждой цепочке собственной и присоединенных функиий $z_{k}^{0}, z_{k}^{1}, \ldots, z_{k}^{h}$, сопряженной задачи, отвечающей собственному значению $\lambda_{k}$, соответствует цепочка собственного и присоединенных функиий $z_{k}^{0}, z_{k}^{1}, \ldots, z_{k}^{h}$, пучка $[A(\bar{\lambda})]^{*}$, отвечающая тому же собственному значению $\lambda_{k}$. При этом первые компоненты әлемента $z_{k}^{s}, s=0,1, \ldots, h$, пучка $[A(\bar{\lambda})]^{*}$ совпадают с әлементом $z_{k}^{s}$.

ДокАЗАТЕЛЬСТВо. Заметим, что формы

$$
U_{1}(y, \lambda), U_{2}(y, \lambda), \ldots, U_{n}(y, \lambda), U_{n+1}^{0}(y), U_{n+2}^{0}(y), \ldots, U_{2 n}^{0}(y)
$$

являются линейно независимьми при любом фиксированном $\lambda$. Действительно, предположим противное. Пусть формы не являются линейно независимыми. С помощью условия $\mathbf{U}$ получим, что формы

$$
U_{1}^{0}(y), U_{2}^{0}(y), \ldots, U_{n}^{0}(y), U_{n+1}^{0}(y), U_{n+1}^{0}(y), \ldots, U_{2 n}^{0}(y)
$$

линейно зависимы. А это противоречит выбору линейных однородных форм

$$
U_{n+1}^{0}(y), U_{n+1}^{0}(y), \ldots, U_{2 n}^{0}(y) .
$$

Таким образом, формы

$$
U_{1}(y, \lambda), U_{2}(y, \lambda), \ldots, U_{n}(y, \lambda), U_{n+1}^{0}(y), U_{n+2}^{0}(y), \ldots, U_{2 n}^{0}(y)
$$


являются линейно независимыми при любом фиксированном $\lambda$. Отсюда и из условий $\mathbf{U}$ и $\mathbf{P}$ следует, что сопряженная к задаче (1), (2) задача имеет следующий вид:

$$
\begin{aligned}
l^{*}(z, \lambda)= & \sum_{\nu=0}^{n} \lambda^{\nu} \sum_{s=\nu}^{n}(-1)^{n-s} \cdot\left(\overline{p_{\nu s}(x)} \cdot z(x)\right)^{(n-s)}=0, \\
U_{j}^{*}(z, \lambda)= & \overline{V_{n+1-j}^{0}(z)}+\sum_{\nu=1}^{m} \lambda^{\nu}\left(\overline{\gamma_{j}^{\nu}(z)}-\xi_{j 1}^{\nu} \cdot \overline{V_{2 n}^{0}(z)}-\cdots\right. \\
& \left.-\xi_{j n}^{\nu} \cdot \overline{V_{n+1}^{0}(z)}\right)=0, \quad j=1,2, \ldots, n .
\end{aligned}
$$

Здесь и далее формы $\overline{\gamma_{1}^{\nu}(z)}, \overline{\gamma_{2}^{\nu}(z)}, \ldots, \overline{\gamma_{n}^{\nu}(z)}$ при $\nu=n+1, n+2, \ldots, m$ тождественно равны нулю. Эти формы введены для сокращения записи.

Введем в рассмотрение пучок операторов $[A(\bar{\lambda})]^{*}$, действующий по формуле

$$
\begin{aligned}
{[A(\bar{\lambda})]^{*} \mathbf{z}=} & \left\{l_{0}^{*}(z) V_{n}^{0}(z) V_{n-1}^{0}(z), \ldots, V_{1}^{0}(z)\right\} \\
& +\lambda \cdot\left\{l_{1}^{*}(z) b_{11}, b_{12}, \ldots, b_{1 n}\right\}+\lambda^{2} \cdot\left\{l_{2}^{*}(z) b_{21}, b_{22}, \ldots, b_{2 n}\right\}+\cdots \\
& +\lambda^{n} \cdot\left\{l_{n}^{*} b_{n 1}, b_{n 2}, \ldots, b_{n n}\right\}+\lambda^{n+1} \cdot\left\{0, b_{n+1,1}, b_{n+1,2}, \ldots, b_{n+1, n}\right\}+\cdots \\
& +\lambda^{m} \cdot\left\{0, b_{m 1}, b_{m 2}, \ldots, b_{m n}\right\}
\end{aligned}
$$

и имеющий область определения

$$
\begin{aligned}
D\left([A(\bar{\lambda})]^{*}\right)=\{ & \mathbf{z}=\left\{z, a_{1}, a_{2}, \ldots, a_{n}\right\}: \mathbf{z} \in W_{2}^{n}, \\
& \left.a_{1}=-V_{2 n}^{0}(z), a_{2}=-V_{2 n-1}^{0}(z), \ldots, a_{n}=-V_{n+1}^{0}(z)\right\} .
\end{aligned}
$$

Здесь $b_{\nu j}, \nu=1,2, \ldots, n,-$ числа, определенные равенствами

$$
b_{\nu j}=\gamma_{j}^{\nu}-\overline{\xi_{1 j}^{\nu}} \cdot V_{2 n}^{0}(z)-\overline{\xi_{2 j}^{\nu}} \cdot V_{2 n-1}^{0}(z)-\cdots-\overline{\xi_{n j}^{\nu}} \cdot V_{n+1}^{0}(z),
$$

a $b_{\nu j}, \nu=n+1, n+2, \ldots, m,-$ это числа, определенные равенствами

$$
b_{\nu j}=-\overline{\xi_{1 j}^{\nu}} \cdot V_{2 n}^{0}(z)-\overline{\xi_{2 j}^{\nu}} \cdot V_{2 n-1}^{0}(z)-\cdots-\overline{\xi_{n j}^{\nu}} \cdot V_{n+1}^{0}(z)
$$

Нетрудно проверить, что

$$
\langle A(\lambda) \mathbf{y}, \mathbf{z}\rangle_{L_{2} \times \mathbb{C}^{2}}=\left\langle\mathbf{y},[A(\bar{\lambda})]^{*}, \mathbf{z}\right\rangle_{L_{2} \times \mathbb{C}^{2}}
$$

Равенство (10) означает, что оператор $[A(\bar{\lambda})]^{*}$ является сужением сопряженного пучка операторов. Операторы $A(\lambda)$ и $[A(\bar{\lambda})]^{*}$ являются отображениями на все пространство $L_{2} \times \mathbb{C}^{n}$. Отсюда и из леммы 2 следует, что пучок операторов $[A(\bar{\lambda})]^{*}$ совпадает с сопряженньп к $A(\lambda)$ пучком операторов.

Если $z^{0}$ - собственная функция задачи (8), (9), отвечающая собственному значению $\lambda_{0}$, то, как следует из определения оператора $[A(\bar{\lambda})]^{*}$, элемент

$$
\mathbf{z}^{0}=\left\{z^{0},-V_{2 n}^{0}\left(z^{0}\right),-V_{2 n-1}^{0}\left(z^{0}\right), \ldots,-V_{n+1}^{0}\left(z^{0}\right)\right\}
$$

из пространства $L_{2} \times \mathbb{C}^{n}$ принадлежит области определения $D\left([A(\bar{\lambda})]^{*}\right)$ сопряженного пучка операторов и является собственной функцией пучка $[A(\bar{\lambda})]^{*}$, отвечающего тому же собственному значению $\lambda_{0}$. 
Верно и обратное: если $\mathbf{z}^{0}$ - собственная функция пучка $[A(\bar{\lambda})]^{*}$, то первая компонента этой функции является собственной функцией сопряженной краевой задачи $(8),(9)$ отвечающей тому же собственному значению $\lambda_{0}$. Далее, если $z^{0}, z^{1}, \ldots, z^{h}$ - цепочка собственных и присоединенных функций краевой задачи $(8),(9)$, отвечающая собственному значению $\lambda_{0}$, то элементы

$$
\begin{aligned}
& \mathbf{z}^{0}=\left\{z^{0},-V_{2 n}^{0}\left(z^{0}\right),-V_{2 n-1}^{0}\left(z^{0}\right), \ldots,-V_{n+1}^{0}\left(z^{0}\right)\right\}, \\
& \mathbf{z}^{1}=\left\{z^{1},-V_{2 n}^{0}\left(z^{1}\right),-V_{2 n-1}^{0}\left(z^{1}\right), \ldots,-V_{n+1}^{0}\left(z^{1}\right)\right\}, \\
& \mathbf{z}^{h}=\left\{z^{h},-V_{2 n}\left(z^{h}\right),-V_{2 n-1}\left(z^{h}\right), \ldots,-V_{n+1}\left(z^{h}\right)\right\}
\end{aligned}
$$

составляют цепочку собственных и присоединенных функций пучка $[A(\bar{\lambda})]^{*}$. И наоборот, первые компоненты собственных и присоединенных функций сопряженного пучка операторов образуют цепочку собственных и присоединенных функций сопряженной краевой задачи. Лемма доказана.

ЗАмЕчаниЕ 2. Используя лемму 2 , нетрудно найти сопряженные к операторам $A_{\nu}$, $\nu=0,1,2, \ldots, m$, и показать, что сопряженньй пучок $[A(\bar{\lambda})]^{*}$ совпадает с пучком сопряженных операторов

$$
A^{*}(\bar{\lambda})=A_{0}^{*}+\lambda A_{1}^{*}+\cdots+\lambda^{n} A_{n}^{*}+\cdots+\lambda^{m} A_{m}^{*}
$$

причем $D\left([A(\bar{\lambda})]^{*}\right)=D\left(A_{0}^{*}\right)$.

Обозначим через $y_{k}^{h}, k=1,2, \ldots ; h=0,1, \ldots, p_{k}$, собственные и присоединенные функции краевой задачи (1), (2), образующие каноническую по Келдышу систему. Здесь $p_{k}$ - длина цепочки собственных и присоединенных функций, отвечающих собственному значению $\lambda_{k}$ краевой задачи $(1),(2)$.

Через $\mathbf{y}_{k}^{h}$ обозначим следующий элемент:

$$
\mathbf{y}_{k}^{h}=\left\{y_{k}^{h}, U_{n+1}^{0}\left(y_{k}^{h}\right), U_{n+2}^{0}\left(y_{k}^{h}\right), \ldots, U_{2 n}^{0}\left(y_{k}^{h}\right)\right\}
$$

a через $\hat{\mathbf{y}}_{k}^{h}$ - производные по Келдышу цепочки длины $m$, построенные по элементам $\mathbf{y}_{k}^{h}$ (cм. [7]).

Пусть $z_{k}^{s}$ - собственные и присоединенные функции сопряженной к $(1),(2)$ краевой задачи, образующие каноническую по Келдышу цепочку (здесь $\left.s=0,1, \ldots, p_{k}\right)$, а $\hat{\mathbf{z}}_{k}^{h}-$ производные по Келдышу цепочки длины $m$, построенные по элементам

$$
\mathbf{z}_{k}^{s}=\left\{z_{k}^{s},-V_{2 n}^{0}\left(z_{k}^{s}\right),-V_{2 n-1}^{0}\left(z_{k}^{s}\right), \ldots,-V_{n+1}^{0}\left(z_{k}^{s}\right)\right\}
$$

Теорема. Пусть задача (1), (2) регулярна и выполнены условия $\mathbf{U} u \mathbf{P}$. Тогда әлемент $\tilde{\mathbf{v}} \in \mathscr{W}_{2, U}^{r}$, әде $r \geqslant \max (\rho-n+1, m-n+q)$, разлагается в ряд (3) единственным образом и коэффичиенты разложения находятся по следующей формуле: 
$c_{k h}=P_{k h} / Q_{k h}$. Здесь величины $P_{k h} u Q_{k h}$ определены выражсениями

$$
\begin{aligned}
P_{k h}= & \sum_{s=0}^{n-1} \sum_{\nu=0}^{n-1-s}\left\langle l_{\nu+1+s}\left(v_{\nu}\right), z_{k}^{p_{k}-h, s}\right\rangle_{L_{2}} \\
& \quad-\sum_{j=1}^{n} \sum_{s=0}^{m-1} \sum_{\nu=0}^{m-1-s} U_{j}^{\nu+s+1}\left(v_{\nu}\right) \cdot \overline{V_{2 n+1-j}^{0}}\left(z_{k}^{p_{k}-h, s}\right), \\
Q_{k h}= & \sum_{s=0}^{n-1} \sum_{\nu=0}^{m-1-s}\left\langle l_{\nu+1+s}\left(y_{k}^{h, \nu}\right), z_{k}^{p_{k}-h, s}\right\rangle_{L_{2}} \\
& \quad-\sum_{j=1}^{n} \sum_{s=0}^{m-1} \sum_{\nu 0}^{m-1-s} U_{j}^{\nu+s+1}\left(y_{k}^{h, \nu}\right) \cdot \overline{V_{2 n+1-j}^{0}}\left(z_{k}^{p_{k}-h, s}\right)
\end{aligned}
$$

или выражениями

$$
\begin{aligned}
P_{k h}= & \sum_{s=0}^{n-1} \sum_{\nu=0}^{n-1-s}\left(v_{s}, l_{\nu+1+s}^{*}\left(z_{k}^{p_{k}-h, \nu}\right)\right)_{L_{2}} \\
& +\sum_{i=1}^{n} \sum_{s=0}^{n-1} \sum_{\nu=0}^{n-1-s} U_{n+i}^{0}\left(v_{s}\right) \cdot \overline{\gamma_{i}^{\nu+1+s}}\left(z_{k}^{p_{k}-h, \nu}\right) \\
& -\sum_{i=1}^{n} \sum_{j=1}^{n} \sum_{s=0}^{n-1} \sum_{\nu=0}^{n-1-s} U_{n+i}^{0}\left(v_{s}\right) \cdot \xi_{n-j+1, i}^{\nu+1+s} \cdot \overline{V_{n+j}^{0}}\left(z_{k}^{p_{k}-h, \nu}\right), \\
Q_{k h}= & \sum_{s=0} \sum_{\nu=0}^{n-1-s}\left(y_{k}^{h, s}, l_{\nu+1+s}^{*}\left(z_{k}^{p_{k}-h, \nu}\right)\right)_{L_{2}} \\
& +\sum_{i=1}^{n} \sum_{s=0}^{n-1} \sum_{\nu=0}^{n-1-s} U_{n+i}^{0}\left(y_{k}^{h, s}\right) \cdot \overline{\gamma_{i}^{\nu+1+s}}\left(z_{k}^{p_{k}-h, \nu}\right) \\
& -\sum_{i=1}^{n} \sum_{j=1}^{n} \sum_{s=0}^{n-1} \sum_{\nu=0}^{n-1-s} U_{n+i}^{0}\left(y_{k}^{h, s}\right) \cdot \xi_{n-j+1, i}^{\nu+1+s} \cdot \overline{V_{n+j}^{0}}\left(z_{k}^{p_{k}-h, \nu}\right) .
\end{aligned}
$$

ДокаЗАТЕЛЬСтво тЕоремЫ. 1. Доказательство формул (11), (12). Доказательство состоит из двух этапов. На первом этапе находятся коэффициенты $\hat{c}_{k} h$ разложений по производньм цепочкам пучка операторов. На втором показьвается, что эти коэффициенты совпадают с коэффициентами $c_{k h}$ из разложения $(3)$.

Первьй этап доказательства. В работе [7] для общего пучка операторов, действующего в гильбертовом пространстве, были получены соотношения биортогональности, связьвающие производные по Келдышу цепочки самого пучка и сопряженного к нему. Приложив этот результат $[7$, с. 154$]$ к рассматриваемому пучку

$$
A(\lambda)=A_{0}+\lambda A_{1}+\cdots+\lambda^{n} A_{n}+\cdots+\lambda^{m} A_{m},
$$

получаем следующие соотношения биортогональности:

$$
\frac{\left\langle G \hat{\mathbf{y}}_{k}^{h}, \hat{\mathbf{z}}_{j}^{s},\right\rangle_{\left(L_{2} \times \mathbb{C}^{n}\right)^{m}}}{\left\langle G \hat{\mathbf{y}}_{k}^{h}, \hat{\mathbf{z}}_{k}^{p_{k}-h}\right\rangle_{\left(L_{2} \times \mathbb{C}^{n}\right)^{m}}}=\delta_{k, j} \cdot \delta_{h, p_{j}-s},
$$


где

$$
G=\left(\begin{array}{cccccc}
A_{1} & A_{2} & A_{3} & \ldots & A_{m-1} & A_{m} \\
A_{2} & A_{3} & A_{4} & \ldots & A_{m} & 0 \\
\vdots & \vdots & \vdots & & \vdots & \vdots \\
A_{m-1} & A_{m} & 0 & \ldots & 0 & 0 \\
A_{m} & 0 & 0 & \ldots & 0 & 0
\end{array}\right)
$$

Из этих соотношений биортогональности следует, что если некоторый элемент $\hat{\mathbf{y}} \in$ $\left(L_{2} \times \mathbb{C}^{n}\right)^{m}$ разлагается в ряд

$$
\hat{\mathbf{y}}=\sum_{k=1}^{\infty} \sum_{h=0}^{p_{k}} \hat{c}_{k h} \hat{\mathbf{y}}_{k}^{h}
$$

то коэффициенты $\hat{c}_{k h}$ находятся по следующей формуле:

$$
\hat{c}_{k h}=\frac{\left\langle G \hat{\mathbf{y}}, \hat{\mathbf{z}}_{k}^{p_{k}-h}\right\rangle_{\left(L_{2} \times \mathbb{C}^{n}\right)^{m}}}{\left\langle G \hat{\mathbf{y}}_{k}^{h}, \hat{\mathbf{z}}_{k}^{p_{k}-h}\right\rangle_{\left(L_{2} \times \mathbb{C}^{n}\right)^{m}}} .
$$

Второй этап доказательства. Согласно работе [6] вектор-функция $\tilde{\mathbf{v}}$ разлагается в ряд по собственньм и присоединенным функциям оператора $H$ :

$$
\tilde{\mathbf{v}}=\sum_{k=1}^{\infty} c_{k h} \tilde{\mathbf{v}}_{k}^{h}
$$

Сходимость ряда в (17) рассматривается в норме пространства $\mathscr{W}_{2}^{r+1}$. Поэтому равенство (17) означает выполнение следующих $n$ равенств:

$$
v_{\nu}=\sum_{k=1}^{\infty} \sum_{h=0}^{p_{k}} c_{k h} y_{k}^{h, \nu}, \quad \nu=0,1, \ldots, n-1
$$

где $y_{k}^{h, \nu}$ - производные по Келдьшу цепочки, построенные по собственным и присоединенным функциям спектральной задачи (1), (2), а сами равенства (18) понимаются как равенства функций в пространстве $W_{2}^{r+1+\nu}$. Из равенств (18) получаем следуюшие разложения:

$$
U_{n+j}^{0}\left(v_{\nu}\right)=\sum_{k=1}^{\infty} \sum_{h=0}^{p_{k}} c_{k h} U_{n+j}^{0}\left(y_{k}^{h, \nu}\right),
$$

где $j=1,2, \ldots, n ; \nu=0,1, \ldots, n-1$.

Выражение $U_{i}^{0}\left(v_{\nu}\right)$ имеет смысл только в том случае, когда порядок соболевского пространства $W_{2}^{k}$, которому принадлежит элемент $v_{\nu}$, на единицу больше порядка формы $U_{i}^{0}(\cdot)$. Именно поэтому в формулировке теоремы требуется, чтобы выполнялось неравенство $r \geqslant m-n+q$.

Максимальный порядок краевых условий $\rho$ не меньше, чем $m$. Поэтому при $m \geqslant n$ имеют смысл следующие равенства:

$$
H \tilde{\mathbf{v}}=H\left(\sum_{k=1}^{\infty} \sum_{h=0}^{p_{k}} c_{k h} \tilde{\mathbf{v}}_{k}^{h}\right)=\sum_{k=1}^{\infty} \sum_{h=0}^{p_{k}} c_{k h} H \tilde{\mathbf{v}}_{k}^{h}=\sum_{k=1}^{\infty} \sum_{h=0}^{p_{k}} c_{k h} \cdot\left(\lambda_{k} \tilde{\mathbf{v}}_{k}^{h}+\tilde{\mathbf{v}}_{k}^{h-1}\right) .
$$


Сходимость рядов в этих равенствах рассматривается уже в норме пространств $\mathscr{W}_{2}^{r}$. Отсюда, в частности, следуют равенства

$$
\begin{aligned}
(H \tilde{\mathbf{v}})_{n-1} & =\sum_{k=1}^{\infty} \sum_{h=0}^{p_{k}} c_{k h} \cdot\left(\lambda_{k} \tilde{\mathbf{v}}_{k}^{h}+\tilde{\mathbf{v}}_{k}^{h-1}\right)_{n-1} \\
& =\sum_{k=1}^{\infty} \sum_{h=0}^{p_{k}} c_{k h} \cdot\left(\lambda_{k} y_{k}^{h, n-1}+y_{k}^{h-1, n-1}\right)=\sum_{k=1}^{\infty} \sum_{h=0}^{p_{k}} c_{k h} y_{k}^{h, n}
\end{aligned}
$$

для $(n-1)$-ой компоненты $(H \tilde{\mathbf{v}})_{n-1}$ вектор-функции $H \tilde{\mathbf{v}}$, понимаемые как равенства в пространстве $W_{2}^{r}$. Здесь $y_{k}^{h, n}-n$-я компонента производной цепочки Келдыша.

Представляя аналогичным образом последние компоненты остальных операторов $H^{i}$, $i=2,3, \ldots, m-n$, получим следуюшие равенства:

$$
\left(H^{i} \tilde{\mathbf{v}}\right)_{n-1}=\sum_{k=1}^{\infty} \sum_{h=0}^{p_{k}} c_{k h} \cdot y_{k}^{h, n+i-1}
$$

понимаемые как равенства в пространстве $W_{2}^{r-i+1}$. Здесь $y_{k}^{h, n+i-1}-(n+i-1)$-я компонента производной цепочки Келдьша.

Из (20) следует, что имеют смысл и верны в пространстве $\mathbb{C}$ комплексных чисел следующие равенства:

$$
U_{n+j}^{0}\left(H^{i} \tilde{\mathbf{v}}\right)_{n-1}=\sum_{k=1}^{\infty} \sum_{h=0}^{p_{k}} c_{k h} U_{n+j}^{0}\left(y_{k}^{h, n+i-1}\right), \quad j=1,2, \ldots, n .
$$

Элементы $\left(H^{i} \tilde{\mathbf{v}}\right)_{n-1}, i=1,2, \ldots, m-n$, будем обозначать через $v_{i+n-1}$. В новых обозначениях равенства (18)-(21) означают, что элемент

$$
\hat{\mathbf{y}}=\left\{\mathbf{v}_{0}, \mathbf{v}_{1}, \ldots, \mathbf{v}_{m-1}\right\}
$$

где $\mathbf{v}_{\nu}=\left\{v_{\nu}, U_{n+1}^{0}\left(v_{\nu}\right), U_{n+2}^{0}\left(v_{\nu}\right), \ldots, U_{2 n}^{0}\left(v_{\nu}\right)\right\}$, разлагается в ряд по элементам

$$
\begin{array}{r}
\hat{\mathbf{y}}_{k}^{h}=\left\{\mathbf{y}_{k}^{h, 0}, \mathbf{y}_{k}^{h, 1}, \ldots, \mathbf{y}_{k}^{h, m-1}\right\}, \\
\text { где } \mathbf{y}_{k}^{h, \nu}=\left\{y_{k}^{h, \nu}, U_{n+1}^{0}\left(y_{k}^{h, \nu}\right), U_{n+2}^{0}\left(y_{k}^{h, \nu}\right), \ldots, U_{2 n}^{0}\left(y_{k}^{h, \nu}\right)\right\}: \\
\hat{\mathbf{y}}=\sum_{k=1}^{\infty} \sum_{h=0}^{p_{k}} c_{k h} \hat{\mathbf{y}}_{k}^{h} .
\end{array}
$$

Равенство (23) понимается как равенство вектор-функций в пространстве

$$
\left(W_{2}^{n+r} \times \mathbb{C}^{n}\right) \times\left(W_{2}^{n+r-1} \times \mathbb{C}^{n}\right) \times \cdots \times\left(W_{2}^{n+r-m} \times \mathbb{C}^{n}\right) .
$$

Так как сходимость в сильной норме влечет за собой сходимость в слабой норме, то сходимость ряда в разложении (23) можно рассматривать в норме пространства $\left(L_{2} \times \mathbb{C}^{n}\right)^{m}$. Но элементы $\hat{\mathbf{y}}_{k}^{h}$ являются производными по Келдышу цепочками пучка 
$A(\lambda)=A_{0}+\lambda A_{1}+\cdots+\lambda^{m} A_{m}$. Поэтому разложение (23) можно рассматривать как разложение элемента $\hat{\mathbf{y}}$ по производным цепочкам Келдьша пучка $A(\lambda)$.

Если в качестве $\hat{\mathbf{y}}$ выбрать элемент $(22)$, то коэффициенты $\hat{c}_{k h}$ являются также коэффициентами $c_{k h}$ разложения (17). Поэтому равенство (16) можно использовать и при вычислении коэффициентов разложения (17). Используя конкретноепредставление для элемента $\hat{\mathbf{y}}$, вычислим коэффициенты $c_{k}$. Для этого найдем сначала скалярное произведение, стоящее в числителе дроби последнего равенства. Имеем

$$
\begin{aligned}
\left\langle G \hat{\mathbf{y}}, \hat{\mathbf{z}}_{k}^{p_{k}-h}\right\rangle_{\left(L_{2} \times \mathbb{C}^{n}\right)^{m}=} & \left\langle A_{1} \mathbf{y}_{0}+A_{2} \mathbf{y}_{1}+\cdots+A_{m} \mathbf{y}_{m-1}, \mathbf{z}_{k}^{p_{k}-h, 0}+\right\rangle_{L_{2} \times \mathbb{C}^{n}} \\
& +\left\langle A_{0} \mathbf{y}_{0}+A_{1} \mathbf{y}_{1}+\cdots+A_{m-1} \mathbf{y}_{m-2}, \mathbf{z}_{k}^{p_{k}-h, 1}+\right\rangle_{L_{2} \times \mathbb{C}^{n}}+\cdots \\
& +\left\langle A_{0} \mathbf{y}_{0}, \mathbf{z}_{k}^{p_{k}-h, m-1}\right\rangle_{L_{2} \times \mathbb{C}^{n}} \\
= & \sum_{s=0}^{m-1} \sum_{\nu=0}^{m-1-s}\left\langle A_{\nu+s+1} \mathbf{y}_{\nu}, \mathbf{z}_{k}^{p_{k}-h, s}\right\rangle_{L_{2} \times \mathbb{C}^{n}} \\
= & \sum_{s=0}^{n-1} \sum_{\nu=0}^{n-1-s}\left\langle l_{\nu+1+s}\left(v_{\nu}\right), z_{k}^{p_{k}-h, s}\right\rangle_{L_{2}} \\
& -\sum_{j=1}^{n} \sum_{s=0}^{m-1} \sum_{\nu=0}^{m-1-s} U_{j}^{\nu+s+1}\left(v_{\nu}\right) \cdot \overline{V_{2 n+1-j}^{0}}\left(z_{k}^{p_{k}-h, s}\right) .
\end{aligned}
$$

Скалярное произведение $\left\langle G \hat{\mathbf{y}}_{k}^{h}, \hat{\mathbf{z}}_{k}^{p_{k}-h}\right\rangle$ вычисляется аналогично. Доказательство формул (11), (12) закончено.

2. Доказательство формул $(13),(14)$. Поскольку операторы $A_{\nu}$ имеют сопряженные, то справедливы следующие равенства:

$$
\hat{c}_{k h}=\frac{\left\langle G \hat{\mathbf{y}}, \hat{\mathbf{z}}_{k}^{p_{k}-h}\right\rangle_{\left(L_{2} \times \mathbb{C}^{n}\right)^{m}}}{\left\langle G \hat{\mathbf{y}}_{k}^{h}, \hat{\mathbf{z}}_{k}^{p_{k}-h}\right\rangle_{\left(L_{2} \times \mathbb{C}^{n}\right)^{m}}}=\frac{\left\langle\hat{\mathbf{y}}, G^{*} \hat{\mathbf{z}}_{k}^{p_{k}-h}\right\rangle_{\left(L_{2} \times \mathbb{C}^{n}\right)^{m}}}{\left\langle\hat{\mathbf{y}}_{k}^{h}, G^{*} \hat{\mathbf{z}}_{k}^{p_{k}-h}\right\rangle_{\left(L_{2} \times \mathbb{C}^{n}\right)^{m}}},
$$

где $G^{*}$ - квадратная матрица размера $m \times m$, составленная из операторов $A_{1}^{*}, A_{2}^{*}, \ldots$, $A_{m-1}^{*}, A_{m}^{*}$ :

$$
G^{*}=\left(\begin{array}{cccccc}
A_{1}^{*} & A_{2}^{*} & A_{3}^{*} & \ldots & A_{m-1}^{*} & A_{m}^{*} \\
A_{2}^{*} & A_{3}^{*} & A_{4}^{*} & \ldots & A_{m}^{*} & 0 \\
\vdots & \vdots & \vdots & & \vdots & \vdots \\
A_{m-1}^{*} & A_{m}^{*} & 0 & \ldots & 0 & 0 \\
A_{m}^{*} & 0 & 0 & \ldots & 0 & 0
\end{array}\right) .
$$

Найдем сначала скалярное произведение, стоящее в числителе дроби из правой части (24). Имеем

$$
\begin{aligned}
& \left\langle\hat{\mathbf{y}}, G^{*} \hat{\mathbf{z}}_{k}^{p_{k}-h}\right\rangle_{\left(L_{2} \times \mathbb{C}^{n}\right)^{m}} \\
& =\left\langle\mathbf{y}_{0}, A_{1}^{*} \mathbf{z}_{k}^{p_{k}-h, 0}+A_{2}^{*} \mathbf{z}_{k}^{p_{k}-h, 1}+\cdots+A_{m}^{*} \mathbf{z}_{k}^{p_{k}-h, m-1}\right\rangle_{L_{2} \times \mathbb{C}^{n}} \\
& \quad+\left\langle\mathbf{y}_{1}, A_{2}^{*} \mathbf{z}_{k}^{p_{k}-h, 0}+A_{3}^{*} \mathbf{z}_{k}^{p_{k}-h, 1}+\cdots+A_{m}^{*} \mathbf{z}_{k}^{p_{k}-h, m-2}\right\rangle_{L_{2} \times \mathbb{C}^{n}}+\cdots \\
& \quad+\left\langle\mathbf{y}_{m-1}, A_{m}^{*} \mathbf{z}_{k}^{p_{k}-h, 0}\right\rangle_{L_{2} \times \mathbb{C}^{n}} \\
& \quad \sum_{s=0}^{m-1}\left\langle\mathbf{y}_{s}, \sum_{\nu=0}^{m-1-s} A_{\nu+1+s}^{*} \mathbf{z}_{k}^{p_{k}-h, \nu}\right\rangle_{L_{2} \times \mathbb{C}^{n}}
\end{aligned}
$$




$$
\begin{aligned}
= & \sum_{s=0}^{n-1} \sum_{\nu=0}^{n-1-s}\left\langle v_{s}, l_{\nu+1+s}^{*}\left(z_{k}^{p_{k}-h, \nu}\right)\right\rangle_{L_{2}} \\
& -\sum_{i=1}^{n} \sum_{j=1}^{n} \sum_{s=0}^{n-1} \sum_{\nu=0}^{n-1-s} U_{n+i}^{0}\left(v_{s}\right) \cdot \xi_{n-j+1, i}^{\nu+1+s} \cdot \overline{V_{n+j}^{0}}\left(z_{k}^{p_{k}-h, \nu}\right) .
\end{aligned}
$$

Скалярное произведение $\left\langle\hat{\mathbf{y}}_{k}^{h}, G^{*} \hat{\mathbf{z}}_{k}^{p_{k}-h}\right\rangle$ вычисляется аналогично. Доказательство теоремы закончено.

ПРИмЕР. При изучении волновых движений жидкости возникает следующая спектральная задача [14]:

$$
y^{\prime \prime}+p(x) y-\lambda^{2} y=0, \quad U_{1}=y(0)=0, \quad U_{2}=y^{\prime}(1)+\left(a+\lambda b-\lambda^{2} c\right) y(1)=0 .
$$

В [15] для этой задачи были вычислены и коэффициенты разложений по производным по Келдьшу цепочкам. Метод, использованньй в этой работе, опирался на построение сопряженного оператора к оператору $H$ и являлся громоздким. Теорема позволяет получить этот же результат проще, непосредственной подстановкой в общую формулу.

Не ограничивая общности (как и в работе [15]), будем считать, что все ее собственные значения являются простыми и нуль не является собственным значением.

Поскольку билинейная форма обынтегрированных членов имеет вид

$$
P_{0}(y, z)=y^{\prime}(1) \overline{z(1)}+y^{\prime}(0) \overline{[-z(0)]}+y(0) \overline{z^{\prime}(0)}+y(1) \overline{\left[-z^{\prime}(1)\right]},
$$

TO

$$
\begin{gathered}
U_{1}^{0}=y(0), \quad U_{2}^{0}=y^{\prime}(1)+a y(1), \quad U_{3}^{0}=y^{\prime}(0), \quad U_{4}^{0}=y(1), \\
V_{4}^{0}=z^{\prime}(0), \quad V_{3}^{0}=z(1), \quad V_{2}^{0}=-z(0), \quad V_{4}^{0}=-z^{\prime}(1)-\bar{a} z(1) .
\end{gathered}
$$

Кроме того,

$$
\begin{gathered}
U_{1}=U_{1}^{0}, \quad U_{2}=U_{2}^{0}+\lambda U_{2}^{1}-\lambda^{2} U_{2}^{2}, \quad U_{2}^{1}=b U_{4}^{0}, \quad U_{2}^{2}=-c U_{4}^{0}, \\
l_{1}^{*}(z)=0, \quad l_{2}^{*}(z)=-z, \quad P_{1}(y, z)=0, \quad P_{2}(y, z)=0,
\end{gathered}
$$

все $\gamma_{i}^{\nu+s+1}$ равны нулю, $\xi_{22}^{1}=b, \xi_{22}^{2}=-c$, остальные $\xi_{i, n-j+1}^{\nu+s+1}$ равны 0.

Из теоремы следует, что $c_{k}=P_{k} / Q_{k}$, где значения $P_{k}$ и $Q_{k}$ можно вычислить по двум формулам. Согласно первой формуле имеем

$$
\begin{aligned}
P_{k}=\left\langle l_{1}\left(v_{0}\right), z_{k}\right\rangle_{L_{2}}+\left\langle l_{2}\left(v_{1}\right), z_{k}\right\rangle_{L_{2}}+\left\langle l_{2}\left(v_{0}\right), \overline{\lambda_{k}} z_{k}\right\rangle_{L_{2}} & \\
& -\sum_{s=0}^{1} \sum_{\nu=0}^{1-s} U_{1}^{\nu+s+1}\left(v_{\nu}\right) V_{5-1}^{0}\left(z_{k}^{0, s}\right)-\sum_{s=0}^{1} \sum_{\nu=0}^{1-s} U_{2}^{\nu+s+1}\left(v_{\nu}\right) V_{5-2}^{0}\left(z_{k}^{0, s}\right) \\
= & -\left\langle v_{1}, z_{k}\right\rangle_{L_{2}}-\lambda_{k}\left\langle v_{0}, z_{k}\right\rangle_{L_{2}} \\
& -b \cdot v_{0}(1) \cdot \overline{z_{k}(1)}+c \cdot v_{1}(1) \cdot \overline{z_{k}(1)}+c \lambda_{k} \cdot v_{0}(1) \cdot \overline{z_{k}(1)} \\
= & -\int_{0}^{1}\left[\lambda_{k} v_{0}(x)+v_{1}(x)\right] \cdot \overline{z_{k}(x)} d x+\left(c \lambda_{k}-b\right) \cdot v_{0}(1) \cdot \overline{z_{k}(1)}+c v_{1}(1) \cdot \overline{z_{k}(1)} \\
Q_{k}= & -2 \lambda_{k} \int_{0}^{1} y_{k}(x) \cdot \overline{z_{k}(x)} d x+2 c \lambda_{k} \cdot y_{k}(1) \cdot \overline{z_{k}(1)}-b y_{k}(1) \cdot \overline{z_{k}(1)} .
\end{aligned}
$$


Согласно второй получаем

$$
\begin{aligned}
P_{k}= & \left\langle v_{0}, l_{2}^{*}\left(\overline{\lambda_{k}} z_{k}\right)\right\rangle_{L_{2}}+\left\langle v_{1}, l_{2}^{*}\left(z_{k}\right)\right\rangle_{L_{2}}+0 \\
& -U_{4}^{0}\left(v_{0}\right) \cdot \xi_{22}^{1} \cdot \overline{V_{3}^{0}\left(z_{k}\right)}-U_{4}^{0}\left(v_{0}\right) \cdot \xi_{22}^{2} \cdot \overline{V_{3}^{0}}\left(\bar{\lambda}_{k}\left(z_{k}\right)\right)-U_{4}^{0}\left(v_{1}\right) \cdot \xi_{22}^{2} \cdot \overline{V_{3}^{0}\left(z_{k}\right)} \\
= & -\int_{0}^{1}\left[\lambda_{k} v_{0}(x)+v_{1}(x)\right] \cdot \overline{z_{k}(x)} d x+\left(c \lambda_{k}-b\right) \cdot v_{0}(1) \cdot \overline{z_{k}(1)}+c v_{1}(1) \cdot \overline{z_{k}(1)} \\
Q_{k}= & -2 \lambda_{k} \int_{0}^{1} y_{k}(x) \cdot \overline{z_{k}(x)} d x+2 c \lambda_{k} \cdot y_{k}(1) \cdot \overline{z_{k}(1)}-b y_{k}(1) \cdot \overline{z_{k}(1)} .
\end{aligned}
$$

Отметим, что коэффициенты вычислены в настоящей работе для разложений в пространстве $\mathscr{W}_{2, U}^{1}$, а в работе [15] для разложений в пространстве $\mathscr{W}_{2, U}^{0} \times \mathbb{C}$.

В заключение автор выражает признательность А. А. Шкаликову за постановку задачи и полезные обсуждения.

\section{СПИСОК ЦИТИРОВАННОЙ ЛИТЕРАТУРЫ}

[1] Birkhoff G. D. On asymptotic character of the solution of certain linear differential equations containing parameter // Trans. Amer. Math. Soc. 1908. V. 9. P. 219-231.

[2] Birkhoff G.D. Boundary value and expansion problems of ordinary linear differential equations // Trans. Amer. Math. Soc. 1908. V. 9. P. 373-395.

[3] Тамаркин Я. Д. О некоторых общих задачах теории обыкновенных дифференциальных уравнений и о разложении произвольных функций в ряды. Петроград, 1917.

[4] Келдыш М. В. О собственных значениях и собственных функциях некоторых классов несамосопряженных уравнений // Докл. АН СССР. 1951. Т. 77. № 1. С. 11-14.

[5] Келдыш М. В. О полноте собственных функций некоторых классов несамосопряженных операторов // УМН. 1971. Т. 26. № 4. С. 15-41.

[6] Шкаликов А. А. Краевые задачи для обыкновенных дифференциальных уравнений с параметром в граничных условиях // Тр. семин. им. И. Г. Петровского. 1983. №9. С. 190-229.

[7] Шкаликов А. А. Эллиптические уравнения в гильбертовом пространстве и спектральные задачи, связанные с ними // Тр. семин. им. И. Г. Петровского. 1989. №14. С. 140-224.

[8] Крылов А.Н. Некоторые замечания о крешерах и индикаторах // Изв. Академии наук. 6 серия. С. -Петербург. 1909. Т. 3. №15. С. 623-654.

[9] Мелешко С. В., Покорный Ю. В. Об одной вибрационной краевой задаче // Дифференц. уравнения. 1987. Т. 23. № 8. С. 1466-1467.

[10] Walter J. Regular eigenvalue problems with eigenvalue parameter in the boundary conditions // Math. Z. 1973. V. 133. №4. P. 301-312.

[11] Hinton D.B. An expansion theorem for an eigenvalue parameter in boundary condition // Quart. J. Math. Oxford. 1979. V. 30. № 2. P. 33-42.

[12] Капустин Н. Ю., Моисеев Е.И. О спектральнй задаче из теории параболо-гиперболического уравнения теплопроводности // Докл. РАН. 1997. Т. 352. № 4. С. 451-454.

[13] Губреев Г. М., Пивоварчик В.Н. Спектральный анализ задачи Редже с параметрами // Функцион. анализ и его прилож. 1997. Т. 31. №1. С. 70-74.

[14] Костюченко А. Г., Шкаликов А. А. К теории самосопряженных квадратичных пучков операторов // Вестн. МГУ. Сер. матем., мех. 1983. №6. С. 40-51.

[15] Ахтямов А. М. О вычислении коэффициентов разложений по производным цепочкам одной спектральной задачи // Матем. заметки. 1992. Т. 51. №6. С. 137-139.

[16] Ахтямов А. М. О коэффициентах разложений по собственным функциям краевой задачи со спектральным параметром в граничных условиях // Изв. вузов. Сер. матем. 2000. № 2. C. $13-18$. 